\title{
Derechos digitales del ciudadano en la gestión de la información
}

\author{
Citizen's digital rights in information management
}

\author{
Vicent Giménez ChoRnet \\ Universitat Politècnica de València. http://orcid.org/0000-0003-1183-9058, vigicho@har.upv.es
}

\begin{abstract}
Resumen
La regulación jurídica de los derechos digitales se inició con la necesidad, en las industrias del entretenimiento, de asegurar los derechos de propiedad intelectual de los autores y editores que producían música o películas. En el presente artículo se analiza cómo en la actualidad se han ampliado los aspectos relacionados con los derechos digitales del ciudadano, como el derecho de control y cesión de datos personales digitales, o los servicios de intermediación en línea.
\end{abstract}

Palabras clave: Derechos digitales. Propiedad intelectual. Datos personales. Servicios de intermediación. Normas jurídicas.

\section{Introducción}

Los derechos digitales irrumpieron en la sociedad de la información, en la primera década de este siglo, como forma de proteger los derechos de autor en las publicaciones librarías. La gestión de los derechos digitales (DRM) aparecieron como una opción de codificar las licencias entre el creador y el editor, y entre el editor y la biblioteca, influyendo en el trabajo del bibliotecario, que debe revisar las cláusulas de la licencia (Böhner 2008). La publicación digital en soportes como CDs o DVDs no garantizó que el contenido digital se pudiese duplicar y distribuir masivamente mediante aplicaciones de intercambio de archivos, que ponían en peligro, especialmente, a las industrias del entretenimiento. Para Kerr y Bailey (2004) hay otros asuntos importantes que plantear, además de la economía de la propiedad intelectual y sus implicaciones financieras en las industrias de la música y la cinematografía, que requiere una comprensión un poco más sólida de los sistemas de gestión de derechos digitales y sus posibles aplicaciones, relacionados con la privacidad personal o con la libertad de expresión, que son parte integral de la democracia como derecho fundamental para la búsqueda de la verdad y para la participación democrática informada. En el entorno de las bibliotecas y de los archivos, con la creación de los archivos digitales, surgió un conflicto de intereses en la gestión de los derechos digitales entre los titulares de

\begin{abstract}
Legal regulation of digital rights began with the need, in the entertainment industries, to secure the intellectual property rights of authors and publishers producing music or films. This article analyses how aspects related to the digital rights of the citizen, such as the right to control and transfer digital personal data, or online intermediation services, have now expanded.
\end{abstract}

Keywords: Digital rights. Intellectual property. Personal data. Intermediation services. Legal rules.

estos derechos, tanto de autor como de propiedad intelectual, frente a los usuarios que se oponían a la posibilidad de que aquello que circulaba por internet estuviese limitado a los que disponían de capacidad de pago, ocasionando una desigualdad y marginación en la población (Ramos Simón, 2002). Esto se consiguió resolver con el impulso de directivas europeas para proteger legalmente las medidas tecnológicas que bloqueaban el acceso a obras si no se disponía de la autorización del titular de los derechos.

Para la gestión de la información digital se desarrollaron estándares y estructuras de metadatos (MARC, METS, Dublin Core, EAD, XrML, o ODRL) que facilitó la gestión integral de los recursos electrónicos, pero ello originó algunos problemas relacionados con la política de los derechos, como el impacto del software propietario, los acuerdos de licencia restrictivos o la gestión de derechos digitales en relación a los requisitos de metadatos que, de alguna manera, debía respaldar el acceso y el uso a largo plazo de la información electrónica (Farb, Riggio, 2004).

En los archivos dependientes de las administraciones públicas, y en los archivos eclesiásticos, aunque sean de titularidad privada, la ley orgánica $3 / 2018$ de protección de datos personales ampara el acceso a los datos e información que estos custodian sobre las personas desaparecidas. La problemática surge en cuanto al tratamiento de los datos personales de estos archivas 
ya que, si bien los archivos de las administraciones públicas hay una supervisión por parte del estado, en el caso del tratamiento de los archivos eclesiásticos, que tienen una estructura muy jerarquizada, no disponen de una autoridad de control, sugiriéndose que debe ser el estado español quien supervise el correcto ejercicio del tratamiento de los datos personales (Corral Sastre, 2016, p. 641; Berrocal Lanzarot, 2016, pp. 816817). Otra problemática en nuestra sociedad de la información en cuanto a los archivos digitales se plantea con la rectificación de los datos personales de las personas fallecidas (García Herrera, 2018), como cuentas bancarias o perfiles en las redes sociales que son gestionados a veces por prestadores de servicios.

Un debate más genérico es sobre si las TIC deben estar subordinadas a los intereses del individuo para preservar su intimidad en nuestra era digital (Rallo Lombarte, 2020).

\section{Objetivos y metodología}

En la actualidad los derechos digitales abarcan más aspectos que los relacionados con la propiedad intelectual, ya que inciden en toda clase de información que pueda generar el ciudadano en su comunidad. Con la irrupción de la información digital generalizada, con la gestión de datos personales, y con el comercio de servicios digitales, el marco legislativo debe abordar distintos aspectos relacionados con los derechos digitales de los ciudadanos y la gestión de la información creada por las administraciones públicas o por las empresas.

El objetivo primordial del presente artículo es el análisis del marco jurídico y de las instituciones que han abordado la problemática de los derechos entre las partes (usuarios y productores de información) para conocer si existen carencias relacionadas con la información que el ciudadano crea en el entorno digital, y cómo repercute en las distintas partes que conviven en la sociedad de la información.

Para ello se analizarán las políticas sobre la digitalización de la sociedad emanadas por los principales organismos internacionales, y posteriormente se analizará la normativa jurídica que aborda la gestión de la información ante los derechos digitales. Nos interesa identificar qué derechos digitales inciden en la gestión de la información, y para ello se analizarán qué aspectos han considerado primordiales los principales organismos internacionales que disponen de una buena reputación en nuestra sociedad. Por otra parte, también se consultarán las sentencias más relevantes para conocer qué aspectos del marco jurídico de los derechos digitales de las partes ha entrado en conflicto en nuestra sociedad.

\section{Contexto}

En la sociedad digital los ciudadanos deben mantener los mismos derechos que ya están reconocidos en convenciones y normativas jurídicas nacionales. La sociedad digital se está implantado en los estados, y organismos supranacionales la potencian. La digitalización ha impulsado una nueva economía donde algunas instituciones del conocimiento relacionadas con la educación, la investigación y el desarrollo desempeñan un papel estratégico en los desafíos de esta nueva sociedad (Cobo Romaní, 2009). Un reciente impulso de la Unión Europea para la generalización de la digitalización parte de las conclusiones del Consejo Europeo de 19 de octubre de 2017. Se apuesta por una Europa más fuerte y coherente, reconociendo que el avance en la digitalización fomenta las oportunidades de innovación, crecimiento, creación de empleo, mejora competitiva, y que favorecerá la diversidad creativa y cultural. Para ello el Consejo Europeo advierte que hay que reconsiderar las políticas que intervienen en la digitalización. Para construir la Europa digital, el Consejo Europeo concluye que la Unión Europea necesita (Consejo Europeo, 2017):

- Administraciones y servicios públicos que hayan hecho una transición completa a la era digital y que prediquen con el ejemplo (la administración electrónica y el despliegue de nuevas tecnologías, la accesibilidad, el punto de acceso general electrónico de la Administración).

- Un marco reglamentario con vocación de futuro (impulsar una estrategia para el Mercado Único Digital).

- Una infraestructura y una red de comunicaciones de primer orden (un medio es implantar redes fijas y móviles de máxima calidad, la tecnología 5G, en toda la UE).

- Un planteamiento común de la ciberseguridad (el mundo digital se basa en la confianza que pasa por una política digital que proporcione una certificación adecuada de seguridad de los productos y servicios, ello facilitará la capacidad para prevenir, disuadir, detectar y responder a los ciberataques).

- Luchar contra el terrorismo y la delincuencia en línea.

- Mercados laborales y sistemas de educación y formación adaptados a la era digital. 
- Hacer un decidido esfuerzo en materia de investigación y desarrollo e inversión (para apoyar nuevas formas de emprendimiento y dar estímulo y ayuda a la transformación digital de industrias y servicios).

- Concienciarse de la urgencia de hacer frente a las nuevas tendencias, lo que comprende cuestiones como la inteligencia artificial y las tecnologías de cadena de bloques, garantizando al mismo tiempo un elevado nivel de protección de los datos, así como los derechos digitales y las normas éticas.

- Un sistema tributario eficaz y justo que se adapte a la era digital.

Las Naciones Unidas han elaborado un informe para crear un proyecto de resolución titulado Las tecnologías de la información y las comunicaciones para el desarrollo sostenible, en diciembre de 2019, en relación con los Objetivos de Desarrollo Sostenible para el 2030. El informe de la comisión de las Naciones Unidas (2019) reconoce que la tecnología está integrada en la ciencia y en la innovación en pro de los ODS, y también señala que:

[...] las tecnologías de la información y las comunicaciones brindan nuevas oportunidades y plantean nuevos desafíos y que existe una apremiante necesidad de superar los grandes obstáculos a que se enfrentan los países en desarrollo para acceder a las nuevas tecnologías, destacando la necesidad de hacer frente a los principales problemas para salvar las brechas digitales, tanto entre los países como dentro de ellos y entre las mujeres y los hombres y entre las niñas y los niños, $y$ de aprovechar las tecnologías de la información y las comunicaciones en pro del desarrollo, y recordando la necesidad de hacer hincapié en la calidad del acceso para salvar las brechas digitales y de conocimientos, utilizando un enfoque multidimensional que incluya la velocidad, la estabilidad, la asequibilidad, el idioma, la capacitación, la creación de capacidad, el contenido local y la accesibilidad para las personas con discapacidad.

El informe destaca la brecha digital que existe entre los países (desarrollados y en desarrollo) y dentro de ellos, donde no está bien resuelta la disponibilidad y asequibilidad de las TIC, ni el acceso universal a Internet. Reconoce que para lograr la igualdad de género es necesario "adaptar las estrategias en materia de ciencia, tecnología e innovación para abordar el empoderamiento de las mujeres y las niñas y reducir las desigualdades". La brecha digital de género afecta especialmente a las mujeres que se encuentran con más obstáculos para poner en marcha proyectos emprendedores (Acosta Velázquez, 2020). La brecha digital también es un factor determinante en el desarrollo del gobierno electrónico, dado que la desigualdad en el acceso a internet y a la información supone un problema grave en la excusión de una gran parte de los ciudadanos (Alomari, Sandhu, Woods, 2014). En el contexto de un mundo globalizado, las TIC promueven el acceso a la información y los conocimientos, uno de los factores que contribuirán al desarrollo de los países menos adelantados, siendo primordial para ello la libre circulación de información y de conocimientos, así como la creación de sistemas jurídicos transparentes, independientes y no discriminatorios. En general, las TIC pueden acelerar el progreso para cumplir con los 17 Objetivos de Desarrollo Sostenible. El entorno normativo y reglamentario de los países puede ser propicio para que las empresas y las administraciones públicas inviertan e innoven para favorecer el acceso universal a Internet, lo que mejorará la educación, el multilingüismo y la preservación de la cultura.

El Foro de Gobernanza de Internet (IGF) se origina a solicitud del secretario general de las $\mathrm{Na}$ ciones Unidas, tras la Cumbre Mundial sobre la Sociedad de la Información, que trató el tema de la gobernanza en Internet, y se celebró en Ginebra en 2003, y en Túnez en 2005. En la Agenda de Túnez, los párrafos del 71 al 80 están dedicados a la creación del Foro (Unión Internacional de Telecomunicaciones, 2006):

72 Solicitamos al Secretario General de las Naciones Unidas, en un proceso abierto e integrador, que convoque para el segundo trimestre de 2006 una reunión del nuevo foro para diálogo sobre políticas de las múltiples partes interesadas (Foro para la Gobernanza de Internet - IGF), con mandato para:

a) debatir temas de políticas públicas relativos a los elementos claves de la gobernanza de Internet, con objeto de contribuir a la sostenibilidad, la solidez, la seguridad, la estabilidad y el desarrollo de Internet;

b) facilitar el diálogo entre organismos que se ocupan de políticas públicas internacionales transversales y relacionadas con Internet, y debatir temas que no se han incluido en el mandato de organismos existentes:

c) facilitar la comunicación con las organizaciones intergubernamentales apropiadas y otras instituciones en temas de su competencia;

d) facilitar el intercambio de información y de mejores prácticas, y en este sentido aprovechar plenamente las competencias de las comunidades académica, científica y técnica;

e) aconsejar a todas las partes interesadas, sugiriendo soluciones y medios para que Internet esté disponible más rápidamente y esté al alcance de un mayor número de personas en los países en desarrollo;

f) Fortalecer y mejorar la participación de las partes interesadas en los mecanismos de gobernanza de 
Internet actuales y/o futuros, en particular los de países en desarrollo;

g) identificar temas emergentes, exponerlos ante los organismos competentes y el público en general, y, en su caso, formular recomendaciones;

h) contribuir a la creación de capacidad para la gobernanza de Internet en países en desarrollo, aprovechando lo más posible los conocimientos y las competencias locales;

i) promover y evaluar permanentemente la materialización de los principios de la CMSI en los procesos de gobernanza de Internet;

j) debatir temas relativos a los recursos críticos de Internet, entre otras cosas;

k) ayudar a encontrar soluciones a los problemas que plantea la utilización correcta o incorrecta de Internet, que son de particular interés para el usuario común;

I) publicar sus actas.

Tras sucesivos mandatos de las Naciones Unidas, el Foro de Gobernanza de Internet continúa activo y sirve para reunir a personas de diversos grupos de partes interesadas como iguales, en debates sobre cuestiones de política pública relacionadas con Internet (Internet Governance Forum, 2020). En el seno del Foro se establecen unas Coaliciones Dinámicas (Dynamic Coalitions, DC) compuestas de grupos abiertos interesados en un problema o conjunto de problemas de gobernanza de Internet. Estas Coaliciones Dinámicas han tratado las siguientes cuestiones: Coalición dinámica sobre accesibilidad y discapacidad (DCAD), Coalición dinámica sobre tecnologías Blockchain (DC-Blockchain), Coalición dinámica sobre seguridad infantil en línea (DCCOS), Coalición dinámica sobre conectividad comunitaria (DC3), Coalición dinámica sobre valores básicos de Internet (DC-CIV), Coalición dinámica sobre problemas de DNS (DC-DNSI), Coalición dinámica sobre género y gobernanza de Internet (DC-Gender), Coalición dinámica sobre enfoques innovadores para conectar a los desconectados (conectar DC a los desconectados), Coalición dinámica en Internet de las cosas (DC-loT), Coalición dinámica sobre derechos y principios de Internet (IRPC), Coalición dinámica sobre la neutralidad de la red (DCNN), Coalición dinámica sobre la responsabilidad de la plataforma (DCPR), Coalición dinámica sobre acceso público en bibliotecas (DC-PAL), Coalición dinámica sobre publicidad (DC-PUB), Coalición dinámica sobre Escuelas de Gobernanza de Internet (DC-SIG), Coalición dinámica en pequeños estados insulares en desarrollo en la economía de Internet (DC-SIDS), Coalición dinámica sobre la sostenibilidad del periodismo y los medios de comunicación (DC-Sustainability), y Coalición de Jóvenes sobre el Gobierno de Internet (YCIG).

La Coalición Dinámica sobre Derechos y Principios en Internet ha elaborado la Carta de Derechos Humanos y Principios en Internet (Internet Governance Forum, 2015), inspirándose, entre otros documentos, en la Carta de la Asociación para el Progreso de las Comunicaciones de Derechos de Internet (APC, 2006). La Carta de la Coalición Dinámica trata sobre el Acceso a Internet; la No discriminación en el acceso, uso y gobernanza en Internet; la Libertad y Seguridad en Internet; el Desarrollo en Internet; el derecho a la libertad de pensamiento, de conciencia y de religión; el derecho a la libertad de reunión y de asociación pacificas; la Privacidad en Internet; la Protección de datos digitales; la Educación en Internet y sobre Internet; el Acceso al Conocimiento y la Cultura en Internet; Los niños e Internet; Internet y el trabajo; la Participación en línea en los asuntos públicos; la Protección de los consumidores en Internet; la Salud y Servicios sociales en Internet; las Soluciones jurídica y Judicial de las actuaciones relacionadas con Internet; la Apropiación de un orden Internacional y social de Internet; y las Obligaciones y responsabilidades; con unas cláusulas sobre la interdependencia de todos los derechos de esta Carta; la no exhaustividad de la Carta y la interpretación de los derechos y libertades expuestos en ella.

La Unión Europea tiene una propuesta de reglamento del Parlamento Europeo y del Consejo por el que se establece el programa Europa Digital para el período 2021-2027 (Unión Europea, 2018). El objetivo primordial de esta propuesta de reglamento es "apoyar la transformación digital de la economía y la sociedad europeas y aportar sus beneficios a los ciudadanos y las empresas europeos". En el Anexo I, sobre las actividades iniciales del programa, se proponen los siguientes objetivos: Objetivo específico 1, Informática de alto rendimiento; Objetivo específico 2, Inteligencia artificial; Objetivo específico 3, Ciberseguridad y confianza; Objetivo específico 4, Competencias digitales avanzadas; y Objetivo específico 5, Despliegue, mejor uso de las capacidades digitales e interoperabilidad.

En España, la Ley Orgánica 3/2018, de 5 de diciembre, centrada en la protección de los datos personales, tiene un apartado dedicado a la garantía de los derechos digitales (España, 2018). En ella se reconoce que "corresponde a los poderes públicos impulsar políticas que hagan efectivos los derechos de la ciudadanía en Internet promoviendo la igualdad de los ciudadanos y de los grupos en los que se integran para hacer posible el pleno ejercicio de los derechos fundamentales en la realidad digital". En España se reconocen los 
siguientes derechos digitales: Derecho a la neutralidad de Internet, Derecho de acceso universal a Internet, Derecho a la seguridad digital, Derecho a la educación digital, Protección de los menores en Internet, Derecho de rectificación en Internet, Derecho a la actualización de informaciones en medios de comunicación digitales, Derecho a la intimidad y uso de dispositivos digitales en el ámbito laboral, Derecho a la desconexión digital en el ámbito laboral, Derecho a la intimidad frente al uso de dispositivos de videovigilancia y de grabación de sonidos en el lugar de trabajo, Derecho a la intimidad ante la utilización de sistemas de geolocalización en el ámbito laboral, Derechos digitales en la negociación colectiva, Protección de datos de los menores en Internet, Derecho al olvido en búsquedas de Internet, Derecho al olvido en servicios de redes sociales y servicios equivalentes, Derecho de portabilidad en servicios de redes sociales y servicios equivalentes, y Derecho al testamento digital.

Para promover el cumplimiento de los derechos digitales, los gobiernos (del Estado y autonómicos) deben realizar políticas de impulso, en concreto un Plan de Acceso a Internet, para superar las brechas digitales, la existencia de espacios de conexión de acceso público y fomentar medidas educativas para que las personas en riesgo de exclusión digital puedan hacer uso de Internet.

\section{La gestión de la información ante los derechos digitales}

Las bibliotecas, los archivos y los centros de documentación son tres de los ámbitos donde más información se gestiona, y esto atañe a los derechos de propiedad intelectual en línea, al control o cesión de datos, y al uso de datos personales por los proveedores de servicios de intermediación en línea. El depósito en repositorios en línea de documentos con propiedad intelectual, o de documentos administrativos actuales o históricos, ha revolucionado la forma en que los usuarios tradicionales consultan estas obras, pasando de la consulta presencial a la consulta en Internet, con descarga de documentos, la generación de copias que se comparten con nuevos canales de distribución, y produciendo datos que podrían ser gestionados para fines que no son los originarios por los que se crearon, y que pueden causar perjuicios en los derechos digitales de las personas. La Ley Orgánica 3/2018 española hace mención a unos derechos digitales concretos, pero da paso a una ampliación de estos, reconociendo en el artículo 79 que todos aquellos "derechos y libertades consagrados en la Constitución y en los Tratados y Convenios Internacionales en que España sea parte son plenamente aplicables en Internet".
4.1. La titularidad y el control de los derechos de propiedad intelectual en línea

La Ley Orgánica 3/2018 española no hace ninguna mención a los derechos de propiedad intelectual en Internet. Reconoce el derecho a la educación digital, y lo relaciona con la protección de datos personales, con la transmisión de valores constitucionales y fundamentales, con la seguridad de los medios digitales, y establece la incorporación de las materias relacionadas con la garantía de los derechos digitales a los temarios de acceso a las administraciones públicas. Pero, en cambio, no existe ninguna referencia en ella a la propiedad intelectual de los contenidos en la educación digital.

La Carta de Derechos Humanos y Principios en Internet del Internet Governance Forum, de las Naciones Unidas; desarrollada por la Coalición Dinámica sobre Derechos y Principios en Internet, sí que establece unas directrices en reconocimiento y para la gestión de los derechos de propiedad intelectual. Defiende el acceso al conocimiento libre de restricciones mediante licencias y copyright, y a la disposición de dominio público de dicho conocimiento. Tras reconocer que todos los creadores deben ser remunerados y reconocidos por su trabajo, se apuesta por lo que denomina un "uso justo" de dichas licencias, que pasa por licencias permisivas, como Creative Commons, que facilitan crear, compartir, utilizar y construir el conocimiento. Ese uso justo que limita las licencias de derecho de autor debe permitir la realización de copias para uso personal y en el aula, los prestamos bibliotecarios, o el uso para la investigación o la sátira. En cuanto a la creación o conocimiento de dominio público se especifica que la investigación y el trabajo intelectual y cultural financiados con fondos públicos deben ser libremente puestos a disposición del público en general, siempre que sea posible (Internet Governance Forum, 2015). Esta Carta hace prevalecer los derechos digitales de los usuarios del conocimiento en línea frente a un derecho expansivo de los derechos de los creadores.

La Ley de Propiedad Intelectual española (España, 1996) protege a las dos partes, a los usuarios de obras en bibliotecas, archivos o museos, y a los creadores. En el artículo 37, dedicado a la reproducción (en muchos casos se da en digital), préstamo y consulta de obras mediante terminales especializados, los usuarios de derechos digitales se beneficiarán en cuanto:

1. Los titulares de los derechos de autor no podrán oponerse a las reproducciones de las obras, cuando aquéllas se realicen sin finalidad lucrativa por los museos, bibliotecas, fonotecas, filmotecas, 
hemerotecas o archivos de titularidad pública o integradas en instituciones de carácter cultural o científico y la reproducción se realice exclusivamente para fines de investigación o conservación.

2. Asimismo, los museos, archivos, bibliotecas, hemerotecas, fonotecas o filmotecas de titularidad pública o que pertenezcan a entidades de interés general de carácter cultural, científico o educativo sin ánimo de lucro, o a instituciones docentes integradas en el sistema educativo español, no precisarán autorización de los titulares de derechos por los préstamos que realicen.

En defensa de los autores, para que no redunde en un perjuicio económico, se aclara en este artículo 37.2 que

[...] los titulares de estos establecimientos remunerarán a los autores por los préstamos que realicen de sus obras en la cuantía que se determine mediante Real Decreto. La remuneración se hará efectiva a través de las entidades de gestión de los derechos de propiedad intelectual.

Solo quedan excluidos de dicha remuneración los establecimientos de titularidad pública en municipios de menos de 5.000 habitantes y las bibliotecas dependientes de instituciones docentes integradas en el sistema educativo español. La remuneración no corre a cargo de los usuarios en los establecimientos de titularidad pública, sino a cargo de las administraciones públicas de las que dependen, y en los casos de los municipios corresponde a las diputaciones provinciales.

La Directiva (UE) 2019/790 del Parlamento Europeo y del Consejo, de 17 de abril de 2019, sobre los derechos de autor y derechos afines en el mercado único digital (Unión Europea 2019a) refuerza que los organismos de investigación de la Unión Europea, combinada o no con la prestación de servicios educativos, y sus bibliotecas, que desarrollan sus actividades sin fines lucrativos (no se refiere a los organismos de investigación que permiten una influencia decisiva de las empresas comerciales, en condición de accionistas o socios), están exentos de pagos por derechos de autor, ratificando la Directiva 2001/29/CE del Parlamento Europeo y del Consejo, de 22 de mayo de 2001, relativa a la armonización de determinados aspectos de los derechos de autor y derechos afines a los derechos de autor en la sociedad de la información (Unión Europea 2001) que en su considerando apunta a que

[...] debe ofrecerse a los Estados miembros la posibilidad de establecer determinadas excepciones o limitaciones en casos tales como aquellos en que se persiga una finalidad educativa o científica, en beneficio de organismos públicos, tales como bibliotecas y archivos, con fines de información periodística, para citas, para uso por personas minusválidas, para usos relacionados con la seguridad pública y para uso en procedimientos administrativos y judiciales.

En este sentido, en el artículo 5, apartado 2, letra c, se estipula que hay exención "en relación con actos específicos de reproducción efectuados por bibliotecas, centros de enseñanza o museos accesibles al público, o por archivos, que no tengan intención de obtener un beneficio económico o comercial directo o indirecto". Esta directiva europea sobre los derechos de autor ha originado un feroz debate, especialmente por los gigantes tecnológicos de Google y Facebook, que ha dividido a la comunidad en general en dos grupos, los titulares de derechos por una parte, y las plataformas de intercambio de contenidos en internet, como puedan ser las bibliotecas, los archivos o las instituciones educativas (Majumdar, 2020).

La puesta a disposición en Internet de copias digitales de obras de autores adquiridas de segunda mano ha suscitado el litigio que ha llegado al Tribunal de Justicia europeo, en este litigio se contraponen los derechos de los autores frente a los derechos digitales de acceso al conocimiento de los usuarios. En 2019 se sentencia el caso holandés de Nederlands Uitgeversverbond (Asociación Editorial Holandesa) y Groep Algemene Uitgevers (Grupo de Editores Generales), demandantes en el litigio principal, como asociaciones que tienen por objeto proteger los intereses comunes de los editores neerlandeses, contra Tom Kabinet Internet BV, demandado en el litigio principal, como sociedad neerlandesa que tiene un sitio de Internet donde proporciona un mercado en línea de libros electrónicos de segunda mano. En concreto, Tom Kabinet revende libros electrónicos, que ha comprado a los distribuidores oficiales y a otros particulares, a los usuarios registrados en su sitio de Internet (https://www.tomka binet.nl/). Los precios ofrecidos por Tom Kabinet son inferiores a los de los distribuidores oficiales (Curia Europea, 2019a). El abogado general del litigio, el Sr. Maciej Szpunar, en sus conclusiones parte de la premisa de que

[...] el agotamiento del derecho de distribución, cuyo origen podemos encontrarlo en la doctrina, fue introducido a principios del siglo $\mathrm{XX}$ por vía jurisprudencial. Según esta regla, una vez que la copia de una obra protegida por derechos de autor ha sido comercializada de forma legal, el titular de los derechos de autor no puede oponerse a la reventa de dicha copia por parte de su adquirente. La justificación de tal regla es que los derechos de autor no pueden prevalecer sobre el derecho de propiedad que ostenta ese adquirente sobre la copia de la obra en cuestión como objeto. Además, se considera que mediante la comercialización de una copia de la obra por parte del autor o con su consentimiento, este ha obtenido la remuneración debida por dicha copia. 
Según este abogado, también en el Derecho de la Unión existe la regla del agotamiento del derecho de distribución de los bienes protegidos por derechos de autor, pero afirma que en el presente caso se plantea que

[...] la digitalización de los contenidos que pueden estar protegidos por derechos de autor y la aparición de nuevos medios para suministrar tales contenidos en línea han subvertido el equilibrio entre los intereses de los titulares de los derechos y los de los usuarios de los bienes protegidos, un equilibrio que la regla del agotamiento del derecho de distribución contribuía a mantener.

El Tribunal de Justicia europeo se enfrenta al hecho de tomar una decisión en un nuevo paradigma, en el que los avances tecnológicos cuestionan la regla del agotamiento del derecho de distribución, establecida en el mundo real de las copias-objetos, a la actual era digital, donde podemos encontrar en Internet copias-archivos digitales.

Tanto el Tratado de la Organización Mundial de la Propiedad Intelectual (OMPI) sobre derecho de autor, como la Directiva 2001/29/CE del Parlamento Europeo y del Consejo, de 22 de mayo de 2001, relativa a la armonización de determinados aspectos de los derechos de autor, consideran que los artistas y autores gozan del derecho exclusivo de autorizar la puesta a disposición del público de la obra original o ejemplares para su primera venta u otra transferencia de propiedad, no agotándose el derecho de distribución hasta que no se realice la primera venta u otro tipo de cesión de la propiedad del objeto por el titular del derecho. Otra cuestión diferente es la reproducción de las obras. La Directiva 2001/29/CE delega en los estados miembros la regulación del "derecho exclusivo a autorizar o prohibir la reproducción directa o indirecta, provisional o permanente, por cualquier medio y en cualquier forma, de la totalidad o parte" (art. 2). Al Tribunal de Justicia europeo se le plantea por el órgano jurisdiccional remitente la cuestión de

[...] si el derecho de distribución en el sentido del artículo 4 de la Directiva 2001/29 se aplica al suministro de libros electrónicos por medio de descargas en línea para uso permanente, a si este derecho se agota como consecuencia de tal suministro realizado con el consentimiento del autor y a si los actos de reproducción necesarios para una eventual transmisión sucesiva de un libro electrónico adquirido de esta forma son legales.

Sin embargo, para el abogado general esta cuestión compleja se centra en (Curia Europea 2019a):

[...] ¿ha de entenderse comprendido en el ámbito del derecho de distribución el suministro a los usuarios de obras protegidas mediante descarga en línea, con la consecuencia del agotamiento de dicho derecho por el suministro original realizado con el consentimiento del autor? En particular, la consideración de que dichos actos se rigen por el derecho de comunicación al público excluye que pueda considerarse que se rigen por el derecho de distribución y viceversa. Por otra parte, no me parece lógico ni adecuado establecer una distinción entre el derecho de distribución y el agotamiento de dicho derecho, dado que ambos conceptos se aplican a los mismos actos. Por último, en la medida en que una reproducción de la obra es indispensable para cualquier transmisión a distancia de un archivo electrónico, la cuestión del derecho de reproducción es inherente a cualquier debate sobre un eventual agotamiento del derecho de distribución en línea.

En conclusión, el abogado general interpreta que en base al artículo 3, apartado 1, y el artículo 4 de la Directiva 2001/29/CE del Parlamento Europeo y del Consejo, de 22 de mayo de 2001, relativa a la armonización de determinados aspectos de los derechos de autor y derechos afines a los derechos de autor en la sociedad de la información, que la descarga en línea de libros electrónicos para un uso permanente es un derecho de comunicación al público en el sentido del artículo 3 , apartado 1 , de esta Directiva, y no la ve como un derecho de distribución en el sentido del artículo 4 de la citada Directiva.

Ante ello el Tribunal de Justicia sentencia el 19 de diciembre de 2019 (Curia Europea, 2019b):

\begin{abstract}
El suministro al público mediante descarga de un libro electrónico para su uso permanente está comprendido dentro del concepto de "comunicación al público" y, más específicamente, del de "puesta a disposición del público de [las] obras [de los autores] de tal forma que cualquier persona pueda acceder a ellas desde el lugar y en el momento que elija", en el sentido del artículo 3, apartado 1, de la Directiva 2001/29/CE del Parlamento Europeo y del Consejo, de 22 de mayo de 2001, relativa a la armonización de determinados aspectos de los derechos de autor y derechos afines a los derechos de autor en la sociedad de la información.
\end{abstract}

El Tribunal de Justicia europeo ha valorado los derechos de autor digitales en la comunicación de las obras en Internet, que son inherentes a la reproducción en línea, y por tanto sujetos a los mismos derechos que las obras impresas.

\subsection{Derecho de control y cesión de datos personales digitales}

La Carta de Derechos Humanos y Principios en Internet considera la protección de los datos digitales como un derecho a la intimidad (apartado 9), consagrado en la Declaración de los Derechos Humanos. La protección de los datos y la identidad digital está también relacionada con la 
protección contra todas las formas de delincuencia, constatada en el apartado número 3 de la Carta sobre Libertad y Seguridad en Internet. La protección de los datos personales digitales implica que los estados deben cumplir las normas internacionales sobre la captura, uso, divulgación y conservación de dichos datos para proteger la privacidad; que los colectores de datos cumplan con una política de transparencia en la recopilación, uso, divulgación y conservación de dichos datos, permitiendo que en último término la persona pueda ejercer el control sobre sus datos personales, de forma que quien exija datos personales deberá solicitar el consentimiento del individuo sobre su contenido, almacenamiento, duración, y sobre unos mecanismos para el acceso, recuperación, corrección o eliminación de los datos. Los datos deben ser borrados cuando se ha cumplido la finalidad por la cual se recopilaron, y si son transmitidos a terceros, los recolectores de datos tienen la obligación de solicitar el consentimiento activo. Para esta Carta es importante que el sistema de protección de datos sea vigilado por una autoridad independiente, que ejerza sus funciones de forma transparente, sin ventajas comerciales o influencia política.

La Ley Orgánica 3/2018 española solamente menciona la protección de datos, en los derechos digitales, en el ámbito de los menores, puntualizando que (art. 92).

[... ] los centros educativos y cualesquiera personas físicas o jurídicas que desarrollen actividades en las que participen menores de edad garantizarán la protección del interés superior del menor y sus derechos fundamentales, especialmente el derecho a la protección de datos personales, en la publicación o difusión de sus datos personales a través de servicios de la sociedad de la información.

La preocupación por la privacidad digital, que se concreta en la angustia de perder el control de la información personal a causa del posible uso secundario por otras partes, es debido al potencial de las amenazas, relacionadas, entre otras, con la vulnerabilidad de los datos financieros entre los consumidores, los datos de ubicación privada filtrados de las aplicaciones de los teléfonos inteligentes, las vulnerabilidades en los datos personales almacenados en la nube, la minería de datos de entidades y corporaciones gubernamentales, o las vulnerabilidades que se puedan dar en los dispositivos conectados a un usuario en el Internet de las Cosas (Elhai, Levine, Hall, 2017). Las TIC proporcionan una mayor recopilación de información privada en línea, así como una mayor capacidad de etiquetar y agregar información personal, por lo que los países democráticos, además de las instituciones públicas y privadas, se preocupan porque se den algunas reglas o técnicas para reforzar el control de los usuarios sobre sus datos. Se ha acuñado el concepto de "cultura de privacidad y confianza" el cual enfatiza en que lidiar con los dilemas de privacidad requiere de un enfoque complejo que combine educación y buenas prácticas con una legislación y una tecnología apropiada, para que los usuarios puedan evaluar y elegir el grado adecuado de privacidad en Internet (Noain-Sánchez, 2016). El papel de los auditores independientes en aquellas organizaciones que recopilan y gestionan datos es necesario para enfrentarse a los riesgos de privacidad y seguridad que emanan del ecosistema digital actual, con las amenazas cibernéticas y las violaciones de datos, para poder dar confianza en que se preservan las condiciones de seguridad (confidencialidad, integridad y disponibilidad), y que estos se controlan adecuadamente (La Torre, et al., 2019).

La referencia a las cookies como dispositivos que instalan en los terminales del usuario consta en la Directiva 2002/58/CE del Parlamento Europeo y del Consejo, de 12 de julio de 2002, relativa al tratamiento de los datos personales y a la protección de la intimidad en el sector de las comunicaciones electrónicas (Unión Europea 2002), que si bien constituye un instrumento legítimo y de gran utilidad en algunos casos, como analizar la efectividad de la publicidad de un sitio web o para verificar la identidad de usuarios partícipes en una transacción en línea, en otros casos supone acceder a una información personal, tras el rastreo de las actividades del usuario o los usuarios de un mismo terminal, que puede suponer una grave intrusión en la intimidad. La instalación de las cookies de los sitios web en el hardware personal requiere el consentimiento informado de los usuarios, y sin embargo muchas empresas no ofrecen a los consumidores la posibilidad de optar por no participar (Sørum, Presthus, 2021). La Directiva 2002/58/CE dejó en manos de los estados miembros la legislación para proteger la confidencialidad de las comunicaciones y de los datos de tráfico asociados a ellas (art. 5.1), pero especificando que (art. 6.3)

el proveedor de un servicio de comunicaciones electrónicas disponible para el público podrá tratar los datos a que se hace referencia en el apartado 1 para la promoción comercial de servicios de comunicaciones electrónicas o para la prestación de servicios con valor añadido en la medida y durante el tiempo necesarios para tales servicios o promoción comercial, siempre y cuando el abonado o usuario al que se refieran los datos haya dado su consentimiento. Los usuarios o abonados dispondrán de la posibilidad de retirar su consentimiento para el tratamiento de los datos de tráfico en cualquier momento. 
En cuanto a la captura de datos de geolocalización, que también invade la privacidad de movimiento de las personas, la Directiva 2002/58/CE establece que aquellos que sean diferentes a los datos de tráfico solamente podrán tratarse si se hacen anónimos o previo consentimiento de los usuarios, obligando al proveedor del servicio a informar a los usuarios, antes de obtener el consentimiento, el tipo de datos de geolocalización distintos a los datos de tráfico que almacenará, para qué finalidad, la duración del tratamiento, y si dichos datos se transmitirán a un tercero (art. 9.1).

Las empresas de telecomunicaciones suministran, en ocasiones, un servicio de correo electrónico que podría ser utilizado para la publicidad de un producto. Cualquier persona física o jurídica podría también obtener de sus clientes la dirección de correo electrónico en el contexto de un servicio. En aras de salvaguardar la voluntad del cliente la Directiva 2002/58/CE establece "la posibilidad de oponerse a dicha utilización de las señas electrónicas en el momento en que se recojan las mismas y, en caso de que el cliente no haya rechazado inicialmente su utilización, cada vez que reciban un mensaje ulterior" (art. 13.2), alentando a los estados miembros a que regulen las medidas adecuadas para que no se permitan las comunicaciones no solicitadas con fines de venta directa. El servicio de correo electrónico en Internet de Google, denominado Gmail, fue la cuestión planteada al Tribunal de Justicia europeo en el caso de Google LLC contra la Bundesrepublik Deutschand (República Federal Alemana) en un procedimiento prejudicial (Curia Europea 2019c)

[...] en relación con la resolución de la Bundesnetzagentur für Elektrizität, Gas, Telekommunikation, Post und Eisenbahnen (Agencia Federal de Redes de Electricidad, Gas, Telecomunicaciones, Correos y Ferrocarril, Alemania) ... por la que se declara que el servicio de correo electrónico Gmail de Google constituye un servicio de telecomunicaciones y en consecuencia se le requiere, bajo pena de multa coercitiva, para que cumpla con su obligación de declaración

En el contexto del derecho de la Unión Europea, la Directiva 98/48/CE del Parlamento Europeo y del Consejo, de 20 de julio de 1998 (derogada por la Directiva (UE) 2015/1535), trata sobre una amplia gama de actividades económicas que tienen lugar en línea, pero (Curia Europea 2019c)

[...] la mayoría de estas actividades no están cubiertas por la presente Directiva por no tratarse total o principalmente del transporte de señales en redes de comunicaciones electrónicas. La telefonía vocal y los servicios de correo electrónico están cubiertos por la presente Directiva. Una misma empresa, por ejemplo, un proveedor de servicios de Internet, puede ofrecer tanto un servicio de comunicaciones electrónicas, tal como el acceso a Internet, como servicios no cubiertos por la presente Directiva, tales como el suministro de contenidos en forma de páginas de Internet.

En base a esta directiva las autoridades nacionales, en su reglamentación, promoverán los intereses de los ciudadanos de la Unión Europea, "contribuyendo a garantizar un alto nivel de protección de los datos personales y de la intimidad". Es manifiesto que Google para transmitir el correo electrónico a su destinatario

[...] utiliza servidores de correo electrónico, que efectúan el procesamiento informático necesario para identificar al servidor de destino mediante el Domain Name System (sistema de nombre de dominio, DNS) y proceder al envío de los paquetes de datos. El itinerario seguido por esos paquetes a través de las diversas redes parciales de Internet, operadas por terceros, es dinámico y puede ser constantemente modificado, sin que el usuario del que partió el envío tenga conocimiento de ello ni pueda controlarlo. En el momento de la recepción, un servidor de destino registra el correo electrónico y lo conserva en un buzón de correo electrónico al que el destinatario puede acceder por distintos medios. El itinerario seguido por los correos electrónicos en Internet puede ser más corto cuando se transmiten entre usuarios del mismo proveedor de servicios.

Google alega en su defensa que la explotación de su propia infraestructura de red como parte de Internet no es relevante a esos efectos, ni de hecho ni de derecho, y que dicha infraestructura se implantó principalmente para prestar servicios "ávidos" en cuanto a datos, como la "búsqueda de Google" y "YouTube", pero no es necesaria para la explotación de Gmail, aunque también se utilice para ello, y dado que es un servicio gratuito que pone a disposición de los usuarios, únicamente se financia en una pequeña proporción gracias a la publicidad exhibida en el sitio de Internet de Gmail. Una de las cuestiones que se plantean es si la aportación activa por parte de los usuarios de los datos personales, $\mathrm{u}$ otros datos, o el registro de dichos datos por el prestador del servicio de otro modo durante la utilización del servicio, puede consistir en una remuneración, ya que genera un interés económico al prestador del servicio. Para el Tribunal de Justicia europeo, en su sentencia del 13 de junio de 2019,

El artículo 2, letra c), de la Directiva 2002/21/CE del Parlamento Europeo y del Consejo, de 7 de marzo de 2002, relativa a un marco regulador común de las redes y los servicios de comunicaciones electrónicas (Directiva marco), en su versión modificada por la Directiva 2009/140/CE del Parlamento Europeo y del Consejo, de 25 de noviembre de 2009 , debe interpretarse en el sentido de que un servicio de correo electrónico en Internet que no incluye el acceso a Internet, como el servicio Gmail prestado por Google LLC, no consiste en su totalidad o principalmente en el transporte de señales a través de 
redes de comunicaciones electrónicas y no constituye, por tanto, un "servicio de comunicaciones electrónicas" en el sentido de dicha disposición.

En la Ley Orgánica 3/2018 española se reconoce como uno de los derechos digitales del ciudadano el derecho al olvido en las búsquedas de Internet. En el art. 93.1 se especifica que

[...] toda persona tiene derecho a que los motores de búsqueda en Internet eliminen de las listas de resultados que se obtuvieran tras una búsqueda efectuada a partir de su nombre los enlaces publicados que contuvieran información relativa a esa persona cuando fuesen inadecuados, inexactos, no pertinentes, no actualizados o excesivos o hubieren devenido como tales por el transcurso del tiempo, teniendo en cuenta los fines para los que se recogieron o trataron, el tiempo transcurrido y la naturaleza e interés público de la información.

Este derecho está relacionado con la repercusión de la Sentencia de 13 de mayo de 2014, Google Spain y Google, C-131/12, en la que el Tribunal declara que (Curia Europea, 2014)

[...] el gestor de un motor de búsqueda está obligado a eliminar de la lista de resultados obtenida tras una búsqueda efectuada a partir del nombre de una persona vínculos a páginas web, publicadas por terceros y que contienen información relativa a esta persona, también en el supuesto de que este nombre o esta información no se borren previa o simultáneamente de estas páginas web, y, en su caso, aunque la publicación en dichas páginas sea en sí misma lícita $[\ldots]$

y que el usuario puede

[...] solicitar que la información de que se trate ya no se ponga a disposición del público en general mediante su inclusión en tal lista de resultados, estos derechos prevalecen, en principio, no sólo sobre el interés económico del gestor del motor de búsqueda, sino también sobre el interés de dicho público en acceder a la mencionada información en una búsqueda que verse sobre el nombre de esa persona.

A raíz de esta sentencia, el Conseil d'État (Consejo de Estado actuando como Tribunal Supremo de lo Contencioso-Administrativo, Francia) realiza una petición de decisión prejudicial al Tribunal de Justicia europeo para que precise el alcance territorial de la retirada de enlaces, y si se puede exigir que dicha retirada se haga a nivel nacional, europeo o mundial. Este hecho vuelve a enfrentar a Google LLC, que se ha subrogado en los derechos de Google Inc. contra Commission nationale de l'informatique et des libertés (CNIL), con intervención de Wikimedia Foundation Inc., Fondation pour la liberté de la presse, Microsoft Corp., Reporters Committee for Freedom of the Press y otros, Article 19 y otros, Internet Freedom Foundation y otros, Défenseur des droits (Curia Europea, 2019d). La Sentencia del Tribunal de Justicia de 24 de septiembre de 2019 dictamina que Google debe realizar la retirada de los resultados de búsqueda a nivel de la Unión Europea (Curia Europea, 2019e):

Los artículos 12, letra b), y 14, párrafo primero, letra a), de la Directiva 95/46/CE del Parlamento Europeo y del Consejo, de 24 de octubre de 1995, relativa a la protección de las personas físicas en lo que respecta al tratamiento de datos personales y a la libre circulación de estos datos, y el artículo 17, apartado 1, del Reglamento (UE) 2016/679 del Parlamento Europeo y del Consejo, de 27 de abril de 2016, relativo a la protección de las personas físicas en lo que respecta al tratamiento de datos personales y a la libre circulación de estos datos y por el que se deroga la Directiva 95/46 (Reglamento general de protección de datos), deben interpretarse en el sentido de que, cuando el gestor de un motor de búsqueda estime una solicitud de retirada de enlaces en virtud de estas disposiciones, estará obligado a proceder a dicha retirada no en todas las versiones de su motor, sino en las versiones de este que correspondan al conjunto de los Estados miembros, combinándola, en caso necesario, con medidas que, con pleno respeto de las exigencias legales, impidan de manera efectiva o, al menos, dificulten seriamente a los internautas que efectúen una búsqueda a partir del nombre del interesado desde uno de los Estados miembros el acceso, a través de la lista de resultados que se obtenga tras esa búsqueda, a los enlaces objeto de la solicitud de retirada.

De esta forma el derecho digital al olvido se hace efectivo en toda la Unión Europea, pero no a nivel mundial. Recientemente, el 10 de marzo de 2020, la Autoridad de Protección de Datos de Suecia ha impuesto una multa de 75 millones de coronas suecas a Google, como operador de motores de búsqueda, por incumplimiento del Reglamento de protección de datos, por no cumplir con sus obligaciones con respecto al derecho a solicitar la exclusión en unos resultados de búsqueda. En mayo de 2015, la Inspección de Datos de Suecia, con el apoyo de la Ley de Datos Personales (1998: 204) y la Directiva de Protección de Datos, inició un caso de supervisión (con el número de archivo 1013-2015) contra Google Inc. cuyo sucesor legal es Google LLC. La revisión fue sobre cómo Google maneja las solicitudes de personas físicas en referencia a que ciertos resultados de búsqueda no deben mostrarse cuando alguien busca su nombre, con el respectivo derecho de solicitud de eliminación. La revisión se realizó a la luz de la sentencia de Google Spain (C-131/12) por la que se declaró que las personas tienen derecho, en algunos casos, a una solicitud de eliminación. En dicha revisión se detectaron 13 quejas recibidas por la Inspección de Datos de Suecia, de personas que creían que Google había negado erróneamente sus respectivas solicitudes de eliminación. La Inspección de Datos instó a 
Google que debía tomar medidas sobre cinco de las quejas antes del 2 de agosto de 2017, y que en los resultados de búsqueda especificados no se mostrase el nombre de un reclamante en este litigio. Tras la aplicación del Reglamento de Protección de Datos el 25 de mayo de 2018, una nueva inspección encontró, el 8 de junio de 2018, que aún se mostraban dos quejas en el resultado de búsqueda (Suecia, 2020).

La minería de datos implica un proceso de explotación de datos personales, entre otros tipos de datos generalmente de carácter comercial, que a sus gestores $u$ organizaciones les redunda en beneficios potenciales, pero pueden colisionar con la disponibilidad y la privacidad de los datos sensibles (Grisold, et al., 2021).

\subsection{Servicios de intermediación en línea}

Los derechos digitales de los ciudadanos implican la vigilancia de los proveedores de los servicios de intermediación en línea o de los motores de búsqueda para detectar los incumplimientos que puedan cometer. Los intermediarios de software pueden usar algoritmos integrados en aplicaciones (motores de búsqueda, agregadores de noticias, consumo de entretenimientos, etc.), que pueden influir en nuestra vida diaria a través de resultados de búsqueda o sistemas de recomendación (Saurwein, Just, Latzer, 2015). La Carta de Derechos Humanos y Principios en Internet también señala, en el punto 17 "Soluciones jurídica y judicial de las actuaciones relacionadas con Internet" que ante el derecho a un juicio justo, es cada vez más común que se viole en el entono de Internet porque las empresas de intermediación realizan un juicio determinando si un contenido es ilegal, e instan a eliminarlo sin la correspondiente orden judicial, y que por tanto "es necesario reiterar que los derechos procesales deben ser respetados, protegidos y cumplidos en Internet tanto como en el entorno offline" (Internet Governance Forum, 2015).

La Ley 34/2002, de 11 de julio, de servicios de la sociedad de la información y de comercio electrónico (España 2002), define que los servicios de intermediación son "la provisión de servicios de acceso a Internet, la transmisión de datos por redes de telecomunicaciones, la realización de copia temporal de las páginas de Internet solicitadas por los usuarios, el alojamiento en los propios servidores de datos, aplicaciones o servicios suministrados por otros y la provisión de instrumentos de búsqueda, acceso y recopilación de datos o de enlaces a otros sitios de Internet" (Anexo, definiciones), y los prestadores de estos servicios tienen obligaciones y responsabilidades. El legislador indicó en los considerandos de esta ley que dichos prestadores del servicio deben colaborar para impedir que determinados servicios o contenidos ilícitos se sigan divulgando. El incumplimiento de esta responsabilidad está sujeto no sólo a normas de orden administrativo, sino también a las de tipo civil o penal (esto dependerá de los bienes jurídicos afectados). Entre los derechos digitales de los ciudadanos o de las empresas que contraten con un proveedor de servicios de intermediación españoles está la de recibir información de este "de forma permanente, fácil, directa y gratuita, sobre los diferentes medios de carácter técnico que aumenten los niveles de la seguridad de la información y permitan, entre otros, la protección frente a virus informáticos y programas espía, y la restricción de los correos electrónicos no solicitados" (art. 12 bis, 1), e igualmente el ciudadano o empresa que haya contratado sus servicios debe estar informado por este de las herramientas existentes para el filtrado y restricción del acceso a determinados contenidos y servicios en Internet no deseados o que puedan resultar nocivos para la juventud y la infancia, pudiendo comunicar dicha información en el sitio web del propio proveedor.

El reciente Reglamento (UE) 2019/1150 del Parlamento Europeo y del Consejo, de 20 de junio de 2019, sobre el fomento de la equidad y la transparencia para los usuarios profesionales de servicios de intermediación en línea (Unión Europea $2019 b$ ) señala en los considerandos la importancia de que las empresas puedan confiar en los servicios de intermediación en línea, dado que entre ellos entablan relaciones comerciales, como las transacciones a través de servicios en línea (de donde se obtienen datos) por lo que los usuarios profesionales dependen cada vez más de tales servicios para llegar a los consumidores. Y precisamente a causa de esta dependencia "los proveedores de los servicios de intermediación en línea a menudo cuentan con una superior capacidad de negociación, lo que les permite, en efecto, actuar unilateralmente de una manera que puede ser injusta y perjudicar a los intereses legítimos de los usuarios profesionales $\mathrm{y}$, de modo indirecto, también de los consumidores de la Unión", repercutiendo, por tanto, en el punto 15 de la Carta de Derechos Humanos y Principios en Internet que trata de la protección del consumidor en Internet, donde se especifica que "el comercio electrónico debe estar regulado para asegurar que los consumidores reciben el mismo nivel de protección que disfrutan en las transacciones no electrónicas". En los considerandos del Reglamento (UE) 2019/1150 se señalan algunos aspectos que influyen en los derechos digitales de los ciudadanos consumidores: 
- Garantizar un ecosistema en línea competitivo, justo y transparente para mejorar la confianza de los consumidores en la economía de las plataformas en línea.

- Los motores de búsqueda en línea actúan como importantes fuentes de tráfico en Internet para las empresas que ofrecen bienes y servicios a los consumidores, y el resultado de búsqueda clasificado de esos bienes y servicios tiene un impacto significativo en la posibilidad de elección de los consumidores $\mathrm{y}$, por tanto, en los resultados comerciales, por lo que "a falta de una relación contractual con estos usuarios, los proveedores de motores de búsqueda en línea pueden, por consiguiente, actuar en efecto de una manera unilateral que puede ser injusta y perjudicar a los intereses legítimos de los usuarios de los sitios web corporativos $\mathrm{y}$, de modo indirecto, también de los consumidores en la Unión."

- Ante las prácticas nocivas de los servicios de intermediación se detecta una ausencia de mecanismos efectivos de reclamación.

- La Unión Europea debe garantizar a los profesionales y a los consumidores un mercado interior en línea que sea equitativo, predecible, sostenible y confiable.

- El motor de búsqueda empleado debe ser neutro desde el punto de vista tecnológico, por lo que en el Reglamento se entiende que también quedan incluidas las búsquedas solicitadas por voz.

- Los proveedores de servicios de intermediación en línea deben establecer en la información general de sus condiciones generales los posibles efectos de la titularidad y control de los derechos de propiedad intelectual.

- Los proveedores de servicios de intermediación en línea o de motores de búsqueda en línea tienen derecho a proteger el funcionamiento y los algoritmos de búsqueda, que tanto efecto causan en la capacidad de elección de los consumidores. Pero para respetar también los intereses de los consumidores estos servicios deben hacer "una descripción general de los parámetros de clasificación principales que permita a los usuarios profesionales y a los usuarios de sitios web corporativos adquirir una comprensión adecuada del funcionamiento de la clasificación en el contexto del uso que hagan de servicios específicos de intermediación en línea o de motores de búsqueda en línea".

La responsabilidad de un operador de mercado electrónico en el uso de palabras clave para efectuar publicidad de la venta de un producto en su plataforma digital, cuando esas palabras clave corresponden a la marca del producto que dispone de su plataforma, vulnera derechos. Este es el caso de L'Oréal contra la plataforma eBay, ya que esta ha comprado esas "palabras clave, como marcas de renombre, en servicios remunerados de referenciación en Internet (como AdWords de Google). La introducción de una palabra clave en el motor de búsqueda hace que aparezca un anuncio con un enlace patrocinado que lleva directamente al mercado electrónico de eBay", y corresponde a los Estados miembros garantizar que los titulares de derechos puedan solicitar requerimientos judiciales contra prestadores intermediarios de servicios utilizados por terceros para infringir dichos derechos (Curia Europea 2010). En las conclusiones del abogado general del Tribunal de Justicia europeo, Sr. Jääskinen, se afirma que "no se discute que eBay ha comprado palabras clave constituidas por las marcas de enlace con objeto de activar, en motores de búsqueda como Google, MSN y Yahoo, enlaces a su propio sitio", lo que le lleva a concluir, en 2010, antes de la posterior normativa europea de protección de datos, que

[...] cuando un tercero ha utilizado los servicios de un intermediario, como un operador de un sitio web, para infringir una marca registrada, el artículo $11 \mathrm{de}$ la Directiva 2004/48/CE del Parlamento Europeo y del Consejo, de 29 de abril de 2004, relativa al respeto de los derechos de propiedad intelectual exige que los Estados miembros garanticen que el titular de la marca pueda obtener un requerimiento judicial efectivo, disuasorio y proporcionado contra el intermediario para impedir la continuación o reiteración de la infracción por dicho tercero. Las condiciones y los procedimientos relativos a dichos requerimientos judiciales se definen en el Derecho nacional.

El Reglamento (UE) 2019/1150 defiende el derecho digital de los usuarios a recibir unas indicaciones sobre la clasificación de los bienes y servicios, generalmente debido a unos resultados de los motores de búsqueda, e incluso qué importancia pueden tener las características de clasificación para los consumidores (art. 5.5). En cuanto a los datos capturados por los servicios de intermediación, estos deben informar a los usuarios profesionales a qué categoría de datos ha accedido de los consumidores (art. 9.2.c).

La Comisión Europea quiere fomentar la elaboración de códigos de conducta que deben realizar los servicios de intermediación en línea, así como las organizaciones o asociaciones que los representen, para alcanzar la correcta aplicación del Reglamento. Este Reglamento ha entrado en vigor el 20 de junio de 2020. En el sector audiovisual una preocupación europea es reforzar la capacidad de los operadores de los estados 
miembros en un mercado muy competitivo donde plataformas en línea de distribución mundial pueden perjudicar la producción de obras digitales europeas (Unión Europea, 2021), y en un mercado digital cada vez más abierto y competitivo la distribución de las obras europeas podría repercutir en una limitación al derecho de acceso a dicha información digital.

\section{Conclusión}

La Unión Europea está siendo líder a nivel mundial en defender ciertos derechos digitales de los ciudadanos en el contexto de la digitalización de los países comunitarios. Es necesario poder conjugar la implantación de los avances tecnológicos con la protección de los derechos digitales de las personas en Internet, y que sean compatibles con los Objetivos de Desarrollo Sostenible.

La Coalición Dinámica sobre Derechos y Principios en Internet, del Foro de Gobernanza de Internet, promovido por las Naciones Unidas, ha elaborado una Carta de Derechos Humanos y Principios en Internet más amplia que los derechos digitales contemplados en la Ley Orgánica 3/2018 española.

En la Unión Europea se están realizado acciones en defensa de los derechos digitales de los ciudadanos que están relacionadas con distintos aspectos de la gestión de la información, especialmente con los derechos de propiedad intelectual en línea, con el control o cesión de datos, y el uso de datos personales, y con la información creada por los proveedores de servicios de intermediación en línea, que pueden vulnerar los derechos de los consumidores

Una de las carencias más importantes de marco jurídico respecto a los derechos digitales de los ciudadanos es el de una regulación ante la indefensión de estos de no poder rechazar en un solo clic la instalación de las cookies, o una vez rechazadas las cookies que el sitio web le consulte de nuevo, o le recuerde, si acepta su instalación.

Por otra parte, la Sentencia de 13 de mayo de 2014 de la Curia Europea respecto al derecho al olvido ha obligado que una empresa de servicios tan poderosa como Google Spain y Google deba modificar su algoritmo de búsqueda para no identificar a la persona que los solicite porque vulnera su intimidad o reputación, siendo los países de la Unión Europea los primeros en proteger este derecho digital del ciudadano.

\section{Referencias}

Acosta Velázquez, S. C.; Pedraza Amador, E. M. (2020). La Brecha Digital de Género como factor limitante del desarrollo femenino. // Boletín Científico INVESTIGIUM de la
Escuela Superior de Tizayuca. 5:10, 22-27. https://doi.org/10.29057/est.v5i10.5281.

Alomari, M.K., Sandhu, K. and Woods, P. (2014). Exploring citizen perceptions of barriers to e-government adoption in a developing country. // Transforming Government: People, Process and Policy. 8:1, 131-150. https://doi.org/10.1108/TG-05-2013-0013.

APC (2006). Carta de APC sobre derechos en internet. Asociación para el Progreso de las Comunicaciones. https://www.apc.org/es/pubs/about-apc/carta-de-apc-sobre-derechos-en-internet.

Berrocal Lanzarot, A. I. (2016). Estidio jurídico-crítico sobre la Ley Orgánica $3 / 2018$, de 5 de diciembre de protección de datos personales y garantía de los derechos digitales. Madrid: Reus..

Böhner, D. (2008). Digital rights description as part of digital rights management: a challenge for libraries. // Library $\mathrm{Hi}$ Tech. 26:4, 598-605. https://doi.org/10.1108/07378830 810920923.

Cobo Romaní, J. C. (2009). El concepto de tecnologías de la información. Benchmarking sobre las definiciones de las TIC en la sociedad del conocimiento. // Zer: Revista de estudios de comunicación. 27, 295-318. https:// ojs.ehu.eus/index.php/Zer/article/view/2636/2182.

Consejo Europeo (2017). Reunión del Consejo Europeo (19 de octubre de 2017) - Conclusiones. http://data.consilium.europa.eu/doc/document/ST-14-2017-INIT/es/pdf.

Corral Sastre, A. (2016). El Reglamento General de Protección de Datos de la Unión Europea y los datos de las Iglesias, confesiones y asociaciones religiosas. // Piñar Mañas, J. L. (dir). Reglamento Genera. de Protección de Datos: Hacia un nuevo modelo europeo de privacidad. Madrid: Reus.

Curia Europea (2010). Conclusiones del abogado general Sr. Jääskinen presentadas el 9 de diciembre de 2010. Asunto C-324/09. ECLI:EU:C:2010:757. http://bit.ly/3bbE5z2.

Curia Europea (2014). Sentencia del Tribunal de Justicia (Gran Sala) de 13 de mayo de 2014, Google Spain y Google, C-131/12. http://curia.europa.eu/juris/document/document.jsf?docid=152065\&doclang $=E S$.

Curia Europea (2019a). Conclusiones del abogado general Sr. Maciej Szpunar presentadas el 10 de septiembre de 2019- Asunto C-263/18- Nederlands Uitgeversverbond, Groep Algemene Uitgevers contra Tom Kabinet Internet BV, Tom Kabinet Holding BV, Tom Kabinet Uitgeverij BVECLI:EU:C:2019:697. http://bit.ly/2TU2cfv.

Curia Europea (2019b). Sentencia del Tribunal de Justicia (Gran Sala) de 19 de diciembre de 2019. Nederlands Uitgeversverbond y Groep Algemene Uitgevers contra Tom Kabinet Internet BV y otros. // Eur Lex. https://eur-lex.europa.eu/legal-content/ES/ALL/?uri=CELEX:62018CJ0263.

Curia Europea (2019c). Sentencia del Tribunal de Justicia (Sala Cuarta) de 13 de junio de 2019 en el asunto C193/18, ECLI:EU:C:2019:498. http://bit.ly/2TWK9p1.

Curia Europea (2019d). Conclusiones del abogado general Sr. Maciej Szpunar presentadas el 10 de enero de 2019, Asunto C-507/17- ECLI:EU:C:2019:15. http://bit.ly/39YV Ykj.

Curia Europea (2019e). Sentencia del Tribunal de Justicia (Gran Sala) de 24 de septiembre de 2019, Asunto C507/17, ECLI:EU:C:2019:772. http://bit.ly/3b6neNI.

Elhai, J.; Levine, J.; Hall, B. (2017). Anxiety about electronic data hacking: Predictors and relations with digital privacy protection behavior. // Internet Research. 27:3, 631-649. https://doi.org/10.1108/IntR-03-2016-0070.

España (1996). Real Decreto Legislativo 1/1996, de 12 de abril, por el que se aprueba el texto refundido de la Ley de Propiedad Intelectual, regularizando, aclarando y 
armonizando las disposiciones legales vigentes sobre la materia. BOE, núm. 97, de 22/04/1996. https:// www.boe.es/eli/es/rdlg/1996/04/12/1/con.

España (2002). Ley 34/2002, de 11 de julio, de servicios de la sociedad de la información y de comercio electrónico. BOE, núm. 166. https://www.boe.es/eli/es/l/2002/07/11/ $34 /$ con.

España (2018). Ley Orgánica 3/2018, de 5 de diciembre, de Protección de Datos Personales y garantía de los derechos digitales. BOE, núm. 294, de 06/12/2018. https://www.boe.es/eli/es/lo/2018/12/05/3/con.

Farb, S. E.; Riggio, A. (2004). Medium or message? A new look at standards, structures, and schemata for managing electronic resources. // Library $\mathrm{Hi}$ Tech. 22:2, 144-152. https://doi.org/10.1108/07378830410524576.

García Herrera, V. (2018). El tratamiento de datos de personas fallecidas. Actualidad civil. 5, 12.

Grisold, T.; Mendling, J.; Otto, M.; vom Brocke, J. (2021). Adoption, use and management of process mining in practice. // Business Process Management Journal. 27:2, 369-387. https://doi.org/10.1108/BPMJ-03-2020-0112.

Internet Governance Forum (2015). Carta de derechos humanos y principios para internet. http://internetrightsandprinciples.org/site/wp-content/uploads/2018/10/IRPC_spanish_1stedition_final.pdf.

Internet Governance Forum (2020). About the IGF. https://intgovforum.org/multilingual/tags/about.

Kerr, I. and Bailey, J. (2004). The implications of digital rights management for privacy and freedom of expression. // Journal of Information, Communication and Ethics in Society. 2:2, 85-95. https://doi.org/10.1108/14779960480 000245.

La Torre, M.; Botes, V.; Dumay, J.; Odendaal, E. (2019). Protecting a new Achilles heel: the role of auditors within the practice of data protection. // Managerial Auditing Journal. 36:2, 218-239. https://doi.org/10.1108/MAJ-03-20181836.

Majumdar, S. (2020). Directive (EU) 2019/790 of the European Parliament and of the Council: overhaul of European Union's copyright rules: a study. // Library Hi Tech News. 37:9, 11-13. https://doi.org/10.1108/LHTN-06-2020-0054.

Naciones Unidas (2019). Las tecnologías de la información y las comunicaciones para el desarrollo sostenible. Asamblea General, A/74/378. https://undocs.org/es/A/74/378.

Noain-Sánchez, A. (2016). "Privacy by default" and active "informed consent" by layers: Essential measures to protect ICT users' privacy. // Journal of Information, Communication and Ethics in Society. 14:2, 124-138. https://doi.org/10.1108/JICES-10-2014-0040.

Rallo Lombarte, A. (2020). Una nueva generación de derechos digitales. // Revista de estudios políticos. 187, 101135. https://doi.org/10.18042/cepc/rep.187.04.

Ramos Simón, F. (2002). La gestion de derechos de autor en entornos digitales, un reto para las bibliotecas y centros públicos de información. // Revista General de Información y Documentación. 12:1, 257-277. https://revistas.ucm.es/index.php/RGID/article/view/RGID0202120247A/10205.
Saurwein, F.; Just, N.; Latzer, M. (2015). Governance of algorithms: options and limitations. // Info. 17:6, 35-49. https://doi.org/10.1108/info-05-2015-0025.

Sørum, H.; Presthus, W. (2021). Dude, where's my data? The GDPR in practice, from a consumer's point of view. // Information Technology \& People. 34:3, 912-929. https://doi.org/10.1108/ITP-08-2019-0433.

Suecia (2020). Tillsyn enligt EU:s dataskyddsförordning 2016/679 - Googles hantering av begäranden om borttagande från dess söktjänster. Datainspektionen, Beslut 2020-03-10. https://www.datainspektionen.se/globalassets/dokument/beslut/2020-03-11-beslut-google.pdf.

Unión Europea (2001). Directiva 2001/29/CE del Parlamento Europeo y del Consejo, de 22 de mayo de 2001, relativa a la armonización de determinados aspectos de los derechos de autor y derechos afines a los derechos de autor en la sociedad de la información. // Diario Oficial de la Unión Europea. L 167, 10-19. http://data.europa.eu/ eli/dir/2001/29/oj.

Unión Europea (2002). Directiva 2002/58/CE del Parlamento Europeo y del Consejo, de 12 de julio de 2002, relativa al tratamiento de los datos personales y a la protección de la intimidad en el sector de las comunicaciones electrónicas. // Diario Oficial de la Unión Europea. L 201, 37-47. https://eur-lex.europa.eu/eli/dir/2002/58/oj.

Unión Europea (2018). Propuesta de Reglamento del Parlamento Europeo y del Consejo por el que se establece el programa Europa Digital para el período 2021-2027. // Eur Lex. https://eur-lex.europa.eu/legal-content/ES/TXT/?uri=CELEX:52018PC0434.

Unión Europea (2019a). Directiva (UE) 2019/790 del Parlamento Europeo y del Consejo de 17 de abril de 2019 sobre los derechos de autor y derechos afines en el mercado único digital y por la que se modifican las Directivas 96/9/CE y 2001/29/CE. // Diario Oficial de la Unión Europea. L 130, 92-125. http://data.europa.eu/eli/dir/2019 /790/oj.

Unión Europea (2019b). Reglamento (UE) 2019/1150 del Parlamento Europeo y del Consejo, de 20 de junio de 2019, sobre el fomento de la equidad y la transparencia para los usuarios profesionales de servicios de intermediación en línea. // Diario Oficial de la Unión Europea. L 186, 57-79. http://data.europa.eu/eli/reg/2019/1150/oj.

Unión Europea (2021). Reglamento (UE) 2021/818 del Parlamento Europeo y del Consejo de 20 de mayo de 2021 por el que se establece el Programa Europa Creativa (2021 a 2027) y por el que se deroga el Reglamento (UE) n.o 1295/2013 (Texto pertinente a efectos del EEE). // Diario Oficial de la Unión Europea. L 189/34. https://eur-lex.europa.eu/eli/reg/2021/818/oj.

Unión Internacional de Telecomunicaciones (2006). Agenda de Túnez para la Sociedad de la Información. https://www.itu.int/net/wsis/docs2/tunis/off/6rev1-es.html.

Enviado: 2020-11-14. Segunda versión: 2021-06-01. Aceptado: 2021-06-16. 